\title{
Fusion of Face and Iris Features for Multimodal Biometrics
}

\author{
Ching-Han Chen and Chia Te Chu \\ Institute of Electrical Engineering, I-Shou University, 1, Section 1, \\ Hsueh-Cheng Rd., Ta-Hsu Hsiang, Kaohsiung County, Taiwan 840, R.O.C \\ pierre@isu.edu.tw, cld123@giga.net.tw
}

\begin{abstract}
The recognition accuracy of a single biometric authentication system is often much reduced due to the environment, user mode and physiological defects. In this paper, we combine face and iris features for developing a multimode biometric approach, which is able to diminish the drawback of single biometric approach as well as to improve the performance of authentication system. We combine a face database ORL and iris database CASIA to construct a multimodal biometric experimental database with which we validate the proposed approach and evaluate the multimodal biometrics performance. The experimental results reveal the multimodal biometrics verification is much more reliable and precise than single biometric approach.
\end{abstract}

Keywords: Multimodal biometrics, face, iris, wavelet probabilistic neural network.

\section{Introduction}

With increasing need for reliable authentication schemes, the demand for high reliable automatic person authentication system is very obvious. Traditional automatic personal identification technologies, which use methods such as Personal Identification Number (PIN), ID card, key, etc., to verify the identity of a person, are no longer considered reliable enough to satisfy the security requirement of person authentication system. Hence biometrics-based person authentication system is gaining more and more attention. Biometrics recognition is the process of automatically differentiating people on the basis of individuality information from their physical or behavioral characteristics like fingerprint, iris, face, voice, and etc.

The biometric recognition can be further divided into two modes: identification and verification. The identification mode is designed for identifying an authorized user when he wants to access a biometric recognition system. The system then attempts to find out whom the biometric feature belongs to, by comparing the query sample with a database of enrolled samples in the hope of finding a match. This is known as a one-to-many comparison. On the other side, the verification mode is a one-to-one comparison in which the recognition system tries to verify an individual's identity. In 
this case, a query sample is captured and compared with the previously enrolled sample. If the two samples match, the biometric system confirms that the applicant is the one that he claims to be. In the paper, we will only focus on the issue of biometric verification. As we know, the identification problem is a typically binary classification problem, i.e. accept or reject.

If people use only a single biometric authentication system, the results obtained are not always good enough. This is due to the fact that the precision of single biometric system is easily affected by the reliability of the sensor used. Besides, the single biometric system has still some domain-specific limitation. For example, accuracy of face recognition is affected by illumination, pose and facial expression and the voiceprint is affected by environment noise. According to the report of the US Congress [1], approximately 2 percent of the population does not have a legible fingerprint, that is, cannot be enrolled into a fingerprint biometric system.

Many multimodal biometrics methods and strategies have been proposed [2-8]. In these works, the fusion of the various biometric features is used to make the unique recognition decision. Aiming at the same issue, we integrate two biometric recognition systems, such as face and iris. The purpose is to improve overall error rate by utilizing as much information as possible from each biometric modality.

We previously proposed a series of single biometric approach which including the face recognition [9-11], speaker recognition [12], and iris recognition [13] based on wavelet transform feature extraction. The proposed algorithms are very efficient and suitable for real-time system. The probabilistic neural network is adopted as a common classifier in these methods.

In the multimodal biometric system, we select the face and iris features for constructing a high reliable biometric system, because the face recognition is friendly and non-invasive whereas iris recognition is the most accurate biometrics to date among all biometrics systems [14].

\section{Face and Iris Feature Extraction}

\subsection{Face Feature Extraction}

In [9-11], we proposed an efficient face feature extraction method. A 2-D face image is transformed into 1-D energy profile signal. The face images as Fig. 1 (a) taken at the Olivetti Research Laboratory (ORL) in Cambridge University, U.K. [9].

Let $\mathrm{G}$ be a face image of size $112 \times 92$

$$
\mathrm{G}=\left[\begin{array}{ccc}
g_{1 \times 1} & \cdots & g_{1 \times 92} \\
\vdots & \ddots & \vdots \\
g_{112 \times 1} & \cdots & g_{112 \times 92}
\end{array}\right]
$$

According to the symmetric property of the face, the horizontal signal can be accumulated as 1-D energy signal as Fig. 1(b).

$$
\mathrm{S}=\left[\begin{array}{c}
s_{1} \\
\vdots \\
s_{112}
\end{array}\right]
$$



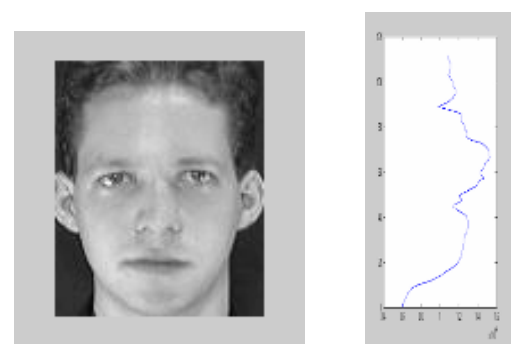

Fig. 1. (a) Facial image (b) 1-D energy signal

\subsection{Iris Feature Extraction}

In [12], we previously proposed a method for low complexity iris feature extraction. Firstly, in order to reduce the computational complexity, we use 2-D wavelet transform to obtain a lower resolution image and localize the pupil position, as shown in Fig.2(a) and (b). By the center of pupil and the radius of pupil, we can acquire the iris circular rings, as shown in Fig.2(c). The more iris circular rings are acquired, the more information is abundant. Secondly, we segment the iris image into three parts and two parts and adopt Sobel transform to enhance iris texture in each part as a feature vector.

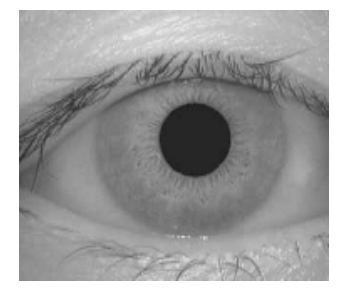

(a)

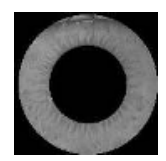

(b)

Fig. 2. Iris location

We extract consecutive circular rings. These circular rings then are stretched horizontally and accumulated, and construct a rectangular-type iris block image, shown as in Fig. 3 (a).

The iris image is divided into three parts, see Fig. 3 (b). The segmented iris image is normalized, see Fig. 3 (d). Subsequently, the Sobel vertical mask

$$
\left[\begin{array}{lll}
-1 & 0 & 1 \\
-2 & 0 & 2 \\
-1 & 0 & 1
\end{array}\right]
$$

is adopted to enhance iris texture in each segmented part, see Fig. 3(d). The purpose of Sobel operator is for enhancing the high frequency signal. 
(a)

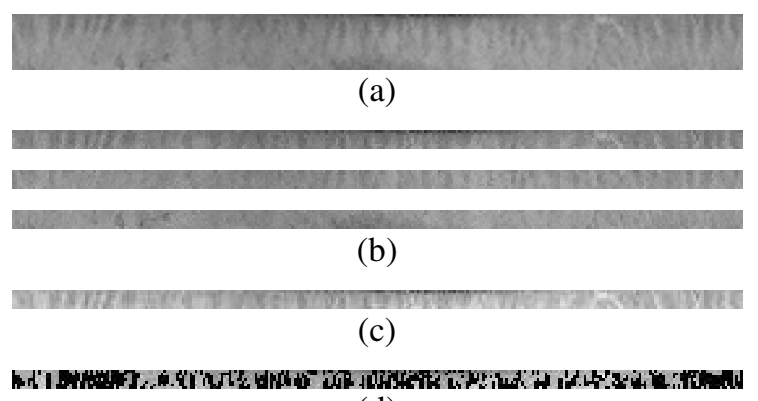

(d)

Fig. 3. Iris feature Extraction: (a) stretched iris block image;(b) iris image divided into three parts; (c) normalized iris image; (d) iris image after Sobel transform

The vertical projection is finally used to convert the block image to 1-D energy profile signal. The projected signal is compact and energy-concentrated. We adopt vertical projection to obtain 1-D energy profile signal and to reduce system complexity. In order to concentrate the energy, every row is accumulated as energy signal.

Let $G$ be a segmented iris image of size $m \times n, m$ is the number of iris circular ring, and $\mathrm{n}$ is pixels of each iris circular ring.

$$
\mathrm{G}=\left[\begin{array}{ccc}
g_{1 x n} & \cdots & g_{1 x n} \\
\vdots & \ddots & \vdots \\
g_{m x 1} & \cdots & g_{m x n}
\end{array}\right]
$$

After vertical projection, the 1-D energy signal $\mathrm{Y}$ is obtained.

$$
\mathrm{S}=\left[\begin{array}{lll}
s_{1} & \cdots & s_{n}
\end{array}\right]
$$

The $\mathrm{m}$ is much smaller than the $\mathrm{n}$. Thus, the information of iris texture after vertical projection is more than the information after horizontal projection.

\section{Wavelet Probabilistic Neural Network (WPNN) Classifier}

The WPNN classifier has been proposed in [11] which is applied for face recognition. Fig. 4 presents the architecture of a four-layer WPNN, which consists of feature layer, wavelet layer, Gaussian layer and decision layer. In feature layer, $X_{1}, \ldots, X_{N}$ are regarded as sets of feature vectors or input data, and $\mathrm{N}$ is the dimension of data sets. The wavelet layer is a linear combination of several multidimensional wavelets. Each wavelet neuron is equivalent to a multidimensional wavelet, and the wavelet in the following form

$$
\phi_{a, b}(x)=\sqrt{a} \phi \frac{x-b}{a} \quad a, b \in R
$$


is a family of function generated from one single function $\phi(x)$ by the scaling and translation, which is localized in both the time space and the frequency space. The $\phi(x)$ is called a mother wavelet and the parameters $\mathrm{a}$ and $\mathrm{b}$ are named respectively scaling factor and translation factor.

In Gaussian layer, the probability density function of each Gaussian neuron is the following form

$$
f_{i}(X)=\frac{1}{(2 \pi)^{\frac{2}{p}} \sigma^{p}}\left(\frac{1}{n_{i}}\right) \sum_{i=1}^{n_{a}} \exp \left(\frac{-\left(X-S_{j}^{i}\right)^{2}}{2 \sigma^{2}}\right)
$$

where $\mathrm{X}$ is the feature vector, $\mathrm{p}$ the dimension of training set, $\mathrm{n}$ the dimension of input data, $\mathrm{j}$ the jth data set, $S_{j}^{i}$ the training set and $\sigma$ the smoothing factor of Gaussian function.

The scaling factor, the translation factor and the smoothing factor are randomly initialized at the beginning and will be trained by PSO algorithm. Once the training is accomplished, the architecture of WPNN and the parameters are fixed for further verification.

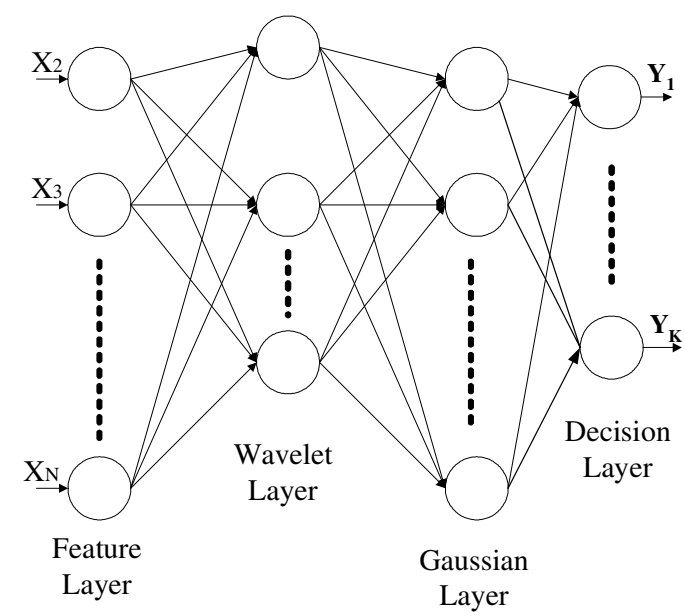

Fig. 4. Wavelet Probabilistic Neural Network

\subsection{Learning Algorithm}

The Particle Swarm Optimization(PSO) is used for training single neuron to optimize WPNN model. PSO is a new bio-inspired optimization method developed by Kenney and Eberhart [15]. The basic algorithm involves the start from a population of distributed individuals, named particles, which tend to move toward the best solution in the search space. The particles will remember the individual best solution encountered 
and the swarm population's best solution. At each iteration, every particle adjusts its velocity vector, based on its momentum and the influence of both its individual best solution and the swarm population's best solution.

At time unit $\mathrm{t}$, the position of $i$ th particle $x_{i}, i=1,2, \ldots, M$, ( $\mathrm{M}$ is the number of particles) moves by adding a velocity vector $v_{i} . v_{i}$ is the function of the best position $p_{i}$ found by that particle, and of the best position $g$ found so far among all particles of the swarm. The movement can be formulated as:

$$
\begin{gathered}
v_{i}=w(t) v_{i}(t-1)+c_{1} u_{1}\left(p-x_{i}(t-1)\right) \\
+c_{2} u_{2}\left(g-x_{i}(t-1)\right) \\
x_{i}(t-1)=x_{i}(t)+v_{i}(t)
\end{gathered}
$$

Where $w(t)$ is the inertia weight, c the acceleration constants, and $\mu \in(0,1)$ the uniformly distributed random numbers.

We encode the wavelet neuron by scaling factor and translation factor of wavelet neuron, and Gaussian neuron by smoothing factor. PSO, in offline mode, searches the best set of factors in the three dimensional space.

\subsection{Decision Rule}

In decision layer of WPNN, There are five inferred probabilistic values $P_{1} P_{2}, \ldots, P_{5}$ for iris features. The average of these five output probabilistic values is $P_{I}$. For face features, there is only one output probabilistic value $P_{f}$. We take the linear

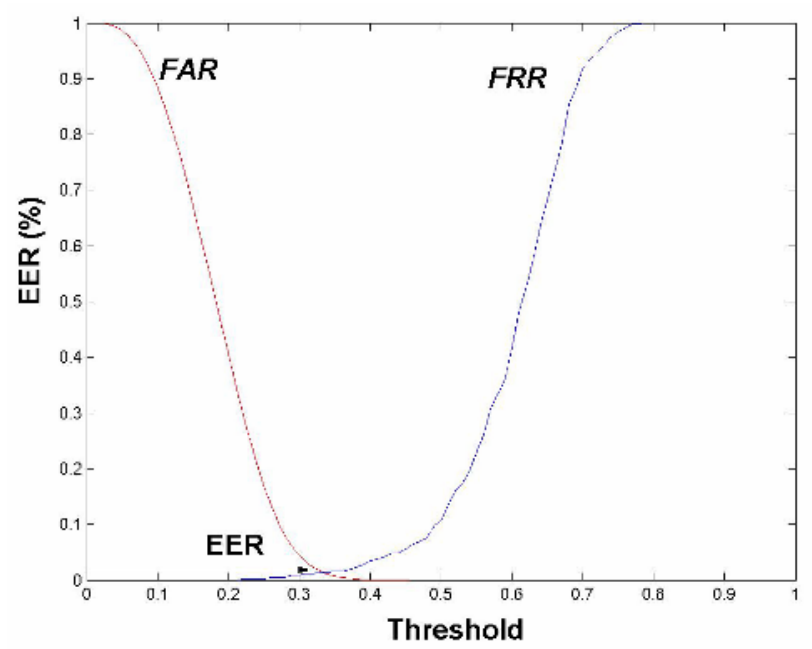

Fig. 5. The curve of FAR-FRR 
combination of these two inference probability, that is the resulting output $P_{a v}$ is the average of $P_{I}$ and $P_{f}$. The fig 5. shows the false rejection ratios (FRR) and false accept ratios (FAR) obtained by adjusting the threshold of $P_{a v}$. The horizontal axis shows the decision threshold of $P_{a v}$ for the multimodal biometrics recognition, and the vertical axis shows each false rate. When the output of $P_{a v}$ for an unregistered sample was lower than the decision threshold, false accepted occurred. The FAR was calculated by counting the trails of false acceptance. On the other hand, when the output of $P_{a v}$ for a resisted sample was higher than the decision threshold, the registered sample was wrong rejected. The FRR was calculated by counting the trials of false rejection.

\section{Experiment and Results}

As the adopted database contains face and iris databases, the face database is from two databases: ORL face database, IIS face database, and the iris database is from CASIA iris database. For the verification experiments, the experiments are divided into two sets. The first set contains 40 subjects from ORL face database and CASIA iris database. The second set contains 100 subjects from IIS face database and CASIA iris database. They are well-known public domain face and iris databases. The ORL database contains 40 subjects and 400 images. The IIS database contains 100 subjects and 3000 images. The CASIA iris database contains 108 subjects and 756 images. The multimodal biometrics recognition system is evaluated in the two sets.

\subsection{Evaluated in ORL Face Database and CASIA Iris Database}

In first set, the multimodal biometric recognition system is evaluated on the ORL face database and CASIA iris database. The face images are sampled from 40 subjects, each subject having 10 images with varying lighting, facial expressions (open / closed eyes, smiling / nonsmiling), facial details (glasses / no glasses) and head pose (tilting and rotation up to 20 degrees). The size of each image is $112 \times 92$. For each subject, the five images are randomly sampled as train sample and the remaining five images as test samples. The CASIA iris database contains 756 iris images acquired of 108 subjects ( 7 images per subject). 40 subjects are randomly selected from CASIA iris database. For each subject, the three images are randomly sampled as train sample and the remaining four images as test samples. Each subject in CASIA iris database is randomly paired with each subject in ORL face database. Such procedures are carried out 100 times. Fig. 7 shows the ROC curve on the three modalities and the experimental results are reported in Table 1.

From the results shown in Fig. 7 and Table 1, they show the recognition performance of different modalities. Fig 7 shows the ROC curves of the three modalities: face, iris, integrated face and iris. From the curves, we can see that the multimodal biometrics 
recognition system achieves better performance than the single face or iris modalities. In Table 1, the multimodal biometrics recognition system has a corresponding best EER of $0.00 \%$ much better than the other two modalities. That is, the multimodal biometrics system is more reliable than single biometrics system.

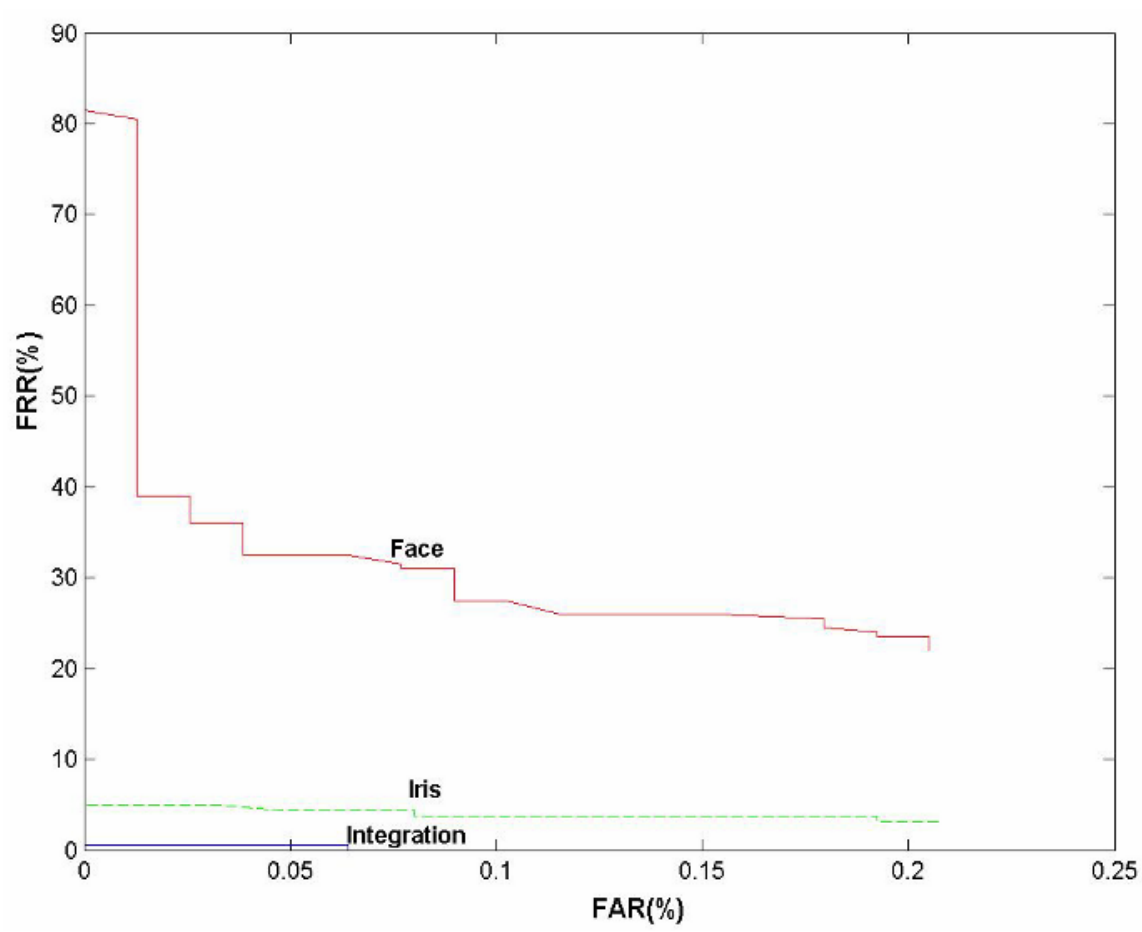

Fig. 7. The ROC curve in different modalities

Table 1. Recognition performance of comparison with different modalities

\begin{tabular}{|c|c|c|c|}
\hline & ORL Face & Iris & Integration \\
\hline Best EER & $1.76 \%$ & $0.02 \%$ & $0.00 \%$ \\
\hline Average EER & $3.83 \%$ & $1.25 \%$ & $0.33 \%$ \\
\hline
\end{tabular}

\subsection{Evaluated in IIS Face Database and CASIA Iris Database}

In second set, the multimodal biometric recognition system is evaluated on the IIS face database and CASIA iris database. The face images are sampled from 100 subjects, each subject having 30 images with varying viewpoints and expressions. The size of each image is $175 \times 155$. For each subject, the six images are randomly sampled as train sample and the remaining twenty-four images as test samples. The algorithm is 
evaluated in the IIS face database. In CASIA iris database, there are 100 subjects randomly selected $\mathrm{f}$. For each subject, the three images are randomly sampled as train sample and the remaining four images as test samples. Each subject in CASIA iris database is randomly paired with each subject in IIS face database. Such procedures are carried out 100 times and the experimental results are reported in Table 2.

Table 2. Recognition performance of comparison with different modalities

\begin{tabular}{|c|c|c|c|}
\hline & IIS Face & Iris & Integration \\
\hline Best EER & $3.59 \%$ & $0.7 \%$ & $0.01 \%$ \\
\hline Average EER & $4.77 \%$ & $1.87 \%$ & $0.64 \%$ \\
\hline
\end{tabular}

Looking at the results shown in Table 2, we can find the multimodal biometric system achieves the best recognition performance among three modalities. The results show further the multimodal biometric modality is better than single biometrics modality. The best EER is $0.01 \%$ and the average EER is 0.64 in the multimodal biometric modality.

\section{Conclusions}

A multimodal biometric system integrating face and iris features is proposed. Firstly, the features of face and iris are separately extracted, and feed into WPNN classifier to make the multimodal decision. We combine a face database ORL and iris database CASIA to construct a multimodal biometric experimental database with which we validate the proposed approach and evaluate the multimodal biometrics performance. The experimental results reveal the multimodal biometrics verification is much more reliable and precise than single biometric approach.

\section{References}

[1] Robert Snelick, Mike Indovina, James Yen, Alan Mink, "Multimodal Biometrics: Issues in Design and Testing", ICMI'03, pp.68-72, British Columbia, Canada, Nov 5-7. 2003.

[2] A. Ross and J. Z. Qian, "Information fusion in biometrics," "in Proc. 3rd International Conference on Audio- and Video-Based Biometric Person Authentication, Halmstad, Sweden, pp. 354-359, June 2001.

[3] A. Ross, A. Jain, "Information fusion in biometrics", Pattern Recognition Letters, vol.24, pp.2115-2125, 2003.

[4] Andrew L. Rukhin, Igor Malioutov, "Fusion of Biometric Algorithm in the Recognition Problem," Pattern Recogition Letters, pp. 299-314, 2001.

[5] R.W. Frischholz and U.Dieckmann,"Bioid: A Multimodal Biometric Identification System", IEEE Computer, vol. 33, pp.64-68, Feb 2000.

[6] C.Sanderson and K.K. Paliwal,'Information Fusion and Person Verification Using Speech \& Face Information", IDIAP, Martigny, Research Report, pp.02-33, 2002. 
[7] Vassilios Chatzis, Adrian G..Bors, and Ioannis Pitas, "Multimodal Decision-Level Fusion for Person Authentication", IEEE Trans. Systems. Man Cybernetics., vol. 29, no. 6, pp.674-680, April. 1999.

[8] Y.Wang, T.Tan and A.K. Jain, "Combining Face and Iris Biometrics for Identity Verification", Proc. Of $4^{\text {th }}$ Int'l Conf. on Audio- and Video-Based Biometric Person Authentication (AVBPA)., pp.805-813, Guildford, UK, June 9-11. 2003.

[9] Chia-Te CHU,Ching-Han CHEN, "The Application of Face Authentication System for Internet Security Using Object-Oriented Technology", Journal of Internet Technology, Special Issue on Object-Oriented Technology and Applications on Internet. (accepted for Oct. 2005 publication)

[10] Ching-Han CHEN, Chia-Te CHU, " Combining Multiple Features for High Performance Face Recognition System ", 2004 International Computer Symposium(ICS2004), pp.387-392,Taipei, Dec 2004.

[11] Ching-Han CHEN, Chia-Te CHU, "Real-Timee Face Recognition Using Wavelet Probabilistic Neural Network", Journal of Imaging Science and Technology. (accepted)

[12] Ching-Han CHEN, Chia-Te CHU, " High Efficiency Iris Feature Extraction Based on 1-D Wavelet Transform ", 2005 Design automation and test in Europe (DATE2005), Munich,Germany, March, 2005.

[13] Ching-Han CHEN, Chia-Te CHU, " An High Efficiency Feature Extraction Based on Wavelet Transform for Speaker Recognition ", 2004 International Computer Symposium(ICS2004), pp. 93-98,Taipei, Dec 2004.

[14] J. Daugman, "How Iris Recognition Works", Proceedings of 2002 International Conference on Image Processing, Vol. 1, 2002.

[15] J. Kennedy, R. Eberhart, "Particle Swarm Optimization," Proc. of IEEE international Conference on Neural Networks (ICNN), vol.IV, pp.1942-1948, Perth, Australia, 1995. 